\title{
Driver Scheduling Problem Modelling
}

\author{
Rita Portugal \\ ICAT - Faculdade de Ciências (FCUL) \\ Universidade de Lisboa, Portugal \\ rita.portugal@icat.fc.ul.pt \\ Helena R. Lourenço \\ GREL - Department of Economics and Business \\ Universitat Pompeu Fabra, Barcelona, Spain \\ helena.ramalhinho@upf.edu \\ José P. Paixão \\ DEIO - Faculdade de Ciências (FCUL) \\ Universidade de Lisboa, Lisboa, Portugal \\ pinto.paixao@cd.fc.ul.pt)
}

\begin{abstract}
The Drivers Scheduling Problem (DSP) consists of selecting a set of duties for vehicle drivers, for example buses, trains, plane or boat drivers or pilots, for the transportation of passengers or goods. This is a complex problem because it involves several constraints related to labour and company rules and can also present different evaluation criteria and objectives. Being able to develop an adequate model for this problem that can represent the real problem as close as possible is an important research area.

The main objective of this research work is to present new mathematical models to the DSP problem that represent all the complexity of the drivers scheduling problem, and also demonstrate that the solutions of these models can be easily implemented in real situations.

The most well-known and general formulation for the DSP is a Set Partition/Set Covering Model (SPP/SCP). However, to a large extend these models simplify some of the specific business aspects and issues of real problems. This makes it difficult to use these models as automatic planning systems because the schedules obtained must be modified manually to be implemented in real situations.

Based on extensive passenger transportation experience in bus companies in Portugal, we propose new alternative models to formulate the DSP problem. These models are also based on Set Partitioning/Covering Models; however, they take into account the bus operator issues and the perspective opinions and environment of the user.

We follow the steps of the Operations Research Methodology which consist of: Identify the Problem; Understand the System; Formulate a Mathematical Model; Verify the Model; Select the Best Alternative; Present the Results of the Analysis and Implement and Evaluate. All the processes are done with close participation and involvement of the final users from different transportation companies. The planner's opinion and main criticisms are used to improve the proposed model in a continuous enrichment process.
\end{abstract}


The final objective is to have a model that can be incorporated into an information system to be used as an automatic tool to produce driver schedules. Therefore, the criteria for evaluating the models is the capacity to generate real and useful schedules that can be implemented without many manual adjustments or modifications. We have considered the following as measures of the quality of the model: simplicity, solution quality and applicability.

We tested the alternative models with a set of real data obtained from several different transportation companies and analyzed the optimal schedules obtained with respect to the applicability of the solution to the real situation. To do this, the schedules were analyzed by the planners to determine their quality and applicability. The main result of this work is the proposition of new mathematical models for the DSP that better represent the realities of the passenger transportation operators and lead to better schedules that can be implemented directly in real situations.

Key-Words: Drivers, Duties, Modelling

\section{Drivers Scheduling Problem}

The Drivers Scheduling Problem (DSP) consists of selecting a set of duties for the vehicle drivers, for example bus, train, plane or boat drivers or pilots, for the transportation of passengers or goods. To evaluate the different alternative models for the DSP we have followed the seven steps of the Operations Research Methodology (Winston 1994). These steps consist on: Identify the Problem; Understand the System; Formulate a Mathematical Model; Verify the Model; Select the Best Alternative; Present the Results of the Analysis and Implement and Evaluate. All the processes are done with close participation and involvement of the final users from different transportation companies, with the main objective to obtain realistic solutions from the models.

In the first step, Identifying the Problem, we start by defining the main objectives of the research which are: To develop mathematical models for the DSP that more closely represent the realities of passenger transportation operators. These models will be incorporated in a Decision Information System, and we hope that the models can produce schedules that can be implemented directly in real situations. In summary, our objective is to take a closer look at the DSP and obtain models and solutions that make business sense to the users.

Although in theory an analyst would hope to include the broad issues and constraints of the problem in the proposed model, a model cannot include every aspect of a situation. A model is always an abstraction that is necessarily a simplification of the real situation; elements that are irrelevant or unimportant to the problem are ignored. The elements considered in the model should leave sufficient detail so that the solution obtained has value with regard to the original problem, which is in this case the real planning process. Models must be both tractable, capable of being solved and valid, representative of the original situation. These dual goals are often contradictory and not always attainable. It is generally true that the most powerful solution methods can be applied to the simplest or most abstract model. Therefore, we are looking for simple but applicable models with high quality solutions.

In the next phase Understand the System, we define the main concepts and collect the sufficient data. The main concepts are explained next. 
The base of the DSP is the Vehicle Schedule that aggregates the need for a driver or crew member for each of several vehicles. A Block is the work of each vehicle from the time that it leaves the depot, or the place were it is parked, until it returns to the same depot or location.

A Relief Point is a place where it is possible to change the driver that is driving a vehicle. A Relief Opportunity is a pair composed of the place and hour where and when it is possible to change the driver that is driving a vehicle. A Work Piece is the period between two consecutive relief's opportunities.

The DSP definition starts with the division of all blocks of vehicles of a schedule in work pieces. If the difference between two non consecutive work pieces is smaller than the break duration it is considerer a Little Interruption. This period of time is usually paid time because, despite he is not driving, the driver is in the vehicle. A Stretch is a consecutive period of vehicle driving formed by several consecutive work pieces or a set of work pieces separated by little interruptions.

A Duty for a driver is a set of stretches that involve the bus driving periods, the breaks and meals periods and the travel of the driver, walking or driving, to the point where the duty or the stretch starts. The duties are defined with a large set of parameters that reflect labour rules, security procedures and planning strategies. These parameters are different depending on the type of duty, and on the number of stretches and breaks permissible within a duty. A Feasible Duty is a duty that meets all the parameters and restrictions that define its type.

The DSP can be defined as: Given a set of work pieces, that result from the division of blocks of a vehicle schedule, obtain the set of feasible duties that guarantee the driving of all vehicles with a minimum cost.

\section{SPP/SCP Models}

In the following three sections we consider next the Winston phase, Formulate the Mathematical Model. We start by presenting the most traditional models for the DSP and discuss the advantages and disadvantages of these models. We then discuss how the users evaluate the solutions of the models on section three, and in the following section we present alternatives models for the DSP which are based on the criticisms and comments obtained by the planners. Given the importance of this phase, we have decided to present it in three sections for clarity.

One of the most common formulations of the DSP is the Set Covering or Set Partitioning Models (SPP/SCP). In the SPP model each work piece is cover by only one duty. In SCP model it is possible to have more that one duty covering each work piece.

In the SPP/SCP models there is a set of work pieces or rows that needs to be covered and a set of previous defined feasible duties or columns that covers specific work pieces. The DSP resolution, based on this two models, is the selection of the feasible duties that guarantee that there is one (SPP) or more (SCP) duties covering each work piece minimizing the total cost of the final schedule.

Consider the SPP/SCP models such that a row represents a work piece and $\mathrm{M}=\{1, \ldots, m\}$ is the set of all rows of the problem. A column represents a previously generated feasible duty and $\mathrm{N}=\{1, \ldots, n\}$ is the set of columns of the problem. 
The coefficients $a_{i j}(i \in \mathrm{M}$ and $j \in \mathrm{N})$ of the constraints matrix are:

$$
a_{i j}= \begin{cases}1 & \text { if the } i \text { work piece is covered by duty } j ; \\ 0 & \text { otherwise; }\end{cases}
$$

The decision variables $x_{\mathrm{j}}(j \in \mathrm{N})$ means:

$$
x_{\mathrm{j}}= \begin{cases}1 & \text { if the duty } j \text { is in the schedule; } \\ 0 & \text { otherwise; }\end{cases}
$$

A solution to the DSP formulated with SPP/SCP models is a vector of decision variables $\boldsymbol{x}=\left(x_{1}, x_{2}, \ldots, x_{n}\right)$, with the value 0 or 1 that specifies if a previously generated feasible duty is on the schedule or not.

The elements $c_{\mathrm{j}}(j \in \mathrm{N})$, coefficients of the objective function, represent the cost of a feasible duty $j$.

Considering the SPP model the DSP has the following formulation:

$$
\begin{array}{lll}
\text { MIN } & \sum_{j=1}^{n} c_{j} x_{j} & \\
\text { s.a: } & \sum_{j=1}^{n} a_{i j} x_{j}=1 & (i \in \mathrm{M}) \\
& x_{j} \in\{0,1\} & (j \in \mathrm{N})
\end{array}
$$

Constraint set (2) guarantees that all work pieces must be covered by only one duty on the schedule. The objective function corresponds to the total cost minimization.

If $c_{\mathrm{j}}=1 \forall j \in \mathrm{N}$ the objective function corresponds to the minimized total number of duties.

Considering the SCP model the DSP has the following formulation:

$$
\begin{array}{lll}
\text { MIN } & \sum_{j=1}^{n} c_{j} x_{j} & \\
\text { S.a: } & \sum_{j=1}^{n} a_{i j} x_{j} \geq 1 & (i \in \mathrm{M}) \\
& x_{j} \in\{0,1\} & (j \in \mathrm{N})
\end{array}
$$

The constraints in set (2) are now inequalities which allow work pieces to be covered by more than one duty on the schedule.

With SCP formulation the final schedule could have some work pieces covered by more than one duty, corresponding to an Overcover Work Piece. In practice this situation corresponds to drivers working to the transportation operators that temporarily do not have any vehicle to drive and is usually called a Reserve Situation. 
One of the biggest advantages of using SPP/SCP models to formulate DSP is the capability of to define two completely different phases during the resolution of the problem; a Generation Phase and a Resolution Phase. The Generation Phase is the previous definition of the set of feasible duties based on the parameters defined by the planners. The Resolution Phase is the selection of a subset of feasible duties for the schedule based on cost minimization. This two phased approach permits the consideration of a big set of different rules when defining feasible duties and facilitates the implementation of the solution methodology with different transportation operators or realities. When changing the bus operator, it is possible to keep all resolution phase methodology and only adapt the generation phase. A big set of different labour rules can be used during feasible duty generation when considering SPP/SCP models. Therefore, the generation phase will obtain duties that verify all impose legislation and labour rules. It is guaranteed that any final schedule obtained in the resolution phase will only have legal duties.

There are a huge number of different rules that a feasible duty should verify like:

- Minimum and maximum stretch duration;

- Minimum and maximum break duration;

- Minimum and maximum work duration;

- Minimum and maximum total duration;

- Maximum extra work duration;

- Maximum number of vehicle changes;

- Minimum driving duration of a particular vehicle.

Despite the simplicity of a general way to define the DSP via the SPP/SCP models, in the real context of the daily business of Portuguese transportation operators, there are several particularities that lead to difficulties in using these simple versions of these models. These characteristics are related to the presence of different evaluation criterias for the schedules and also with the overcover and undercover situations.

With respect to the evaluation criteria, the problems and difficulties associated with SPP/SCP models start with the need for considering desired rules and alternative evaluation criteria during the analysis of final schedules. The desired rules are not imposed by legislation; it is possible to have feasible duties on the schedule that do not verify it, but their implementation facilitates the scheduling needs and the daily scheduling needs and desires of workers.

Some of possible desired rules are:

- Total (Normal Work + Extra Work) duty duration;

- Vehicle changes number;

- Relief's out of the depot;

- Duty type percentage. 
The usual way of considering these rules in the SPP/SCP formulation is by reflecting them in duty cost which represents the real cost plus a penalty cost associated with not verifying these rules. Therefore, the cost parameter $c_{\mathrm{j}}$ beyond representing the real cost of a feasible duty $j$ also reflects the property of the duty associated with the above rules. The penalties values defined by the users should guarantee that duties with desired characteristics have less cost than the ones that do not. Despite the easy and intuitive definition of the concept of penalties, these values are very difficult to adjust. Each individual penalty is difficult to define but the adjustment of a set of different penalties at the same time, for different types of duties, is almost impossible, at least in the reality of a transportation operator. Also, small variations of the values of the penalties lead to very different solutions obtained after solving the SPP/SCP model. The main complaint of planners is that they have difficulty adjusting the penalties such that the cost function represents the appropriate and realistic evaluation criteria. Discussing with them how they evaluate a schedule, by showing them several schedules and ask them to tell us which are "better," we came to the conclusion that they use several evaluation criteria such as the real cost which is obvious, but also the number of duties, the number of trippers and the ones related with the number and duration of over and under cover work pieces. In next section, we discuss in detail these evaluation criteria.

With respect to the undercover and overcover situations, the majority of planners reveal some flexibility in these types of situations. The ideal schedule only has one driver covering each piece of work in the problem, however in most situations this is not feasible. On the other hand, if the number of overcover work pieces is not restricted as in the SCP model, we can have an excess of overcover for some pieces, which leads to a schedule that cannot be implemented and the planner must change it manually. Therefore, a desired property of the "best" schedules are to ones in which the overcover situations are limited and some undercover situations are allowed, so that the planner has some flexibility to change the schedule if desired.

In the beginning of the joint work with these companies, we presented the results of SPP/SCP approaches to several transportation operators' users which are scheduling analysis specialists and asked them to analyse them and make suggestion for improvement. They commented that they would like to have a set of alternative schedules, analyse them, make some manual adjustments and then decide which final schedule that they want to consider and implement. The users have no interest in having only one schedule even it is the optimal solution of SPP/SCP model. They want to consider several active objectives and analyse the best solution obtained for each of them. Also, they mentioned that in general they need to perform some manual changes related to elements that are difficult to include in the model or make the model intractable. Examples of these elements include traffic changes, special work pieces during soccer games or other events, requests by the drivers on a special day, etc.. Ultimately the planners commented that having schedules with work pieces not covered (undercover) and a limited number of overcovered work pieces will facilitate their job when they have to manually modify the schedules. The difficulty of incorporating some specific elements during the manual changes of the optimal solution obtained from the SPP/SCP models creates a situation in which users do not use these models nor the system associated at all. Users in this situation prefer to build the solution in a simple constructive way from scratch. Therefore, one of the planners major requirements when using solutions via any model is that the solutions obtained by the model should be good enough to implement in reality, or be easy to modify such that some elements not included in the model can be considered. 
The SPP/SCP models present good measures with respect of simplicity and solution's quality, however they lack some aspects of applicability as explained above. Therefore, the aim of this work is to develop alternative models to the DSP incorporating the main criticisms and expectations of some Portuguese transportation operators' planners. In the next section we discuss the evaluation criteria and in the following section we present the alternative models.

\section{Evaluation Criteria’s}

The objective function of the SPP/SCP models is to minimize cost. The way this cost function is defined makes an important impact on the solutions obtained. As mentioned before, in many applications of the SPP/SCP models, the cost function has two main parts: the real cost in a monetary value and some penalty costs reflecting the desired rules. The first one is easy to understand by the planner, but the second one presents several difficulties during the process of setting costs. During the close work with the planners, they always comment on this issue because on many occasions these penalties do not make sense to them and they could not assign the appropriate value to the penalties. However, the value of these penalties makes a significant impact on the optimal solutions obtained, see Portugal (2006) for details. Therefore, one of the main issues on the applicability of the SPP/SCP models is the use of penalties on the objective function, and the models presented here try to overcome this difficulty. We propose a set of different evaluation criterias of the schedules related to three main objectives: total "real” cost, undercover work by number of pieces and undercover work duration of pieces. These evaluation criteria more closely reflect the way the planners evaluate a schedule.

Consider the following notations:

- $H I_{i}$-represents the $i^{\text {th }}$ work piece start hour $(i \in \mathrm{M})$;

- $H F_{i}$ - represents the $i^{\text {th }}$ work piece ending hour $(i \in \mathrm{M})$;

- $d_{i}=H F_{i}-H I_{i}-$ represents the $i^{\text {th }}$ work piece duration $(i \in \mathrm{M})$;

- $w_{\mathrm{i}}=\sum_{j=1}^{n} a_{i j} x_{j}$ - represents the $i^{\text {th }}$ number of duties that cover $i$ work piece in the $\boldsymbol{x}$ schedule $(i \in \mathrm{M})$;

The evaluation criteria's of a schedule $\boldsymbol{x}$ are:

(1) Total "real” cost $=\sum_{j=1}^{n} c_{j} x_{j}$;

Several planners consider that is easier to adjust schedules with some undercover work pieces than to analyse and adjust schedules with too many duties and lots of overcover work pieces. In this situation it is feasible to have undercover work pieces but there number should be minimal, since final schedules impose a driver covering each work piece.

(2) Total number of undercover pieces of work $=\sum_{i=1}^{m} \max \left(1-w_{i}, 0\right)$; 
(3) Total duration of undercover pieces of work $=\sum_{i=1}^{m} d_{i} \times \max \left(1-w_{i}, 0\right)$;

The alternative models that will be defined to formulate the new proposed DSP permit undercover work pieces; the criterias (2) and (3) allow control of the number and properties of the undercover situation.

Even in transportation operators that maintain drivers in reserve, the planners are alert to the difficulty of managing the situation of having drivers being paid without driving. Therefore it is important to control the total number or the total duration of drivers in this situation. This is reflected in the following functions:

(4) Total number of overcover work pieces $=\sum_{i=1}^{m} \max \left(\min \left(w_{i}-1,1\right), 0\right)$;

(5) Total duration of overcover work pieces $=\sum_{i=1}^{m} d_{i} \times \max \left(w_{i}-1,0\right)$;

(6) Maximum number of duties involved in the same overcover work piece = $\max _{i \in M}\left(\max \left(w_{i}-1,0\right)\right)$;

In the alternative models that will be defined to formulate the DSP that admit overcover work pieces, the criteria's (4), (5) e (6) allow control over the number and duration of overcover situations.

(7) Unfit $=\sum_{i=1}^{m}\left|w_{i}-1\right|$;

Because the ideal schedules only have one driver covering each work piece of the problem Chu e Beasley (1995), suggest criteria (7) as a good measurement of feasibility of the constraints $\sum_{j=1}^{n} a_{i j} x_{j}=1$.

(8) Number of duties $=\sum_{j=1}^{n} x_{j}$;

This is one of the most important measurements of schedules quality. The personnel costs in a transportation operator are very important and one of the biggest involved in the service. The number of available drivers is not always enough for the daily operations. Good schedules must always have a minimum number of duties.

(9) Trippers number;

Some companies, during the planning process prefer to include the constraint that all work pieces must be covered by some duty. However, in general this leads to infeasible solutions or to many overcover solution. The general procedure in this situation is to consider Trippers. A Tripper is a duty that covers one and only one work piece. In practice the trippers correspond to extra or voluntary work and involve additional costs. A good schedule does not have trippers. Therefore, an important objective function is to minimized the number of tripper used in a solution.

Next we combine these objective functions with some realistic constraints to develop new mathematical models for the DSP. 


\section{Alternative Models to the DSP based on SPP/SCP}

When developing a model for an operational research problem, Hilier and Lieberman (1995) proposed that it should start as a very simple version that evolves to a more complex and real one better reflecting the managers' perspective. This process, named Model Enrichment was considered during this research in addition to some modelling concept present in Ravindran et al. (1987) work.

Because one of the most important measurements of schedule quality is the total number of duties and because the penalty values are so difficult to adjust, our first change to the classic SPP/SCP models, in a monobjective approach, is to consider as the objective function the minimization of the total number of duties (named $\mathrm{SPP}_{\mathrm{Num}} / \mathrm{SCP}_{\mathrm{Num}}$ models). Moreover because, even in transportation operators that permit drivers in a reserve situation, is necessary to control the number and duration of overcover situations to avoid having too many duties covering the same work piece at the same time. In this alternative approach, consider the number of duties covering each work piece is limited by a constant named Maxc. Maxc represents the maximum number of duties involved in the same work piece. The planners must define this parameter, but contrary to the previously mentioned penalties, this parameter has a realistic meaning to the planner so he or she can easily decide on its value. The planners assign a value to this parameter based on their experience, and usually is not higher than three.

The fist proposed model for the DSP, designated by SCP $_{\text {Num_Maxc, has the following }}$ formulation:

$\left(\mathrm{SCP}_{\text {Num_Maxc }}\right)$

$$
\begin{array}{cc}
\text { MIN } \sum_{j=1}^{n} x_{j} & \\
\text { s.a: } \sum_{j=1}^{n} a_{i j} x_{j} \geq 1 & (i \in \mathrm{M}) \\
\sum_{j=1}^{n} a_{i j} x_{j} \leq \operatorname{Maxc} & (i \in \mathrm{M}) \\
x_{j} \in\{0,1\} & (j \in \mathrm{N})
\end{array}
$$

Constraints set (2) guarantees that all work pieces are covered by at least one duty and constraint set (3) guarantees that work pieces are covered by at most Maxc duties for the feasible schedules.

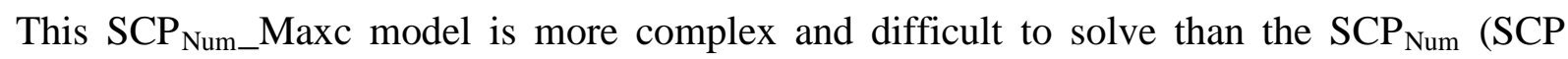
minimizing the total duties number) because of the additional set of constraints. However it includes some aspects that answer to the practical need of the planner, for one side the model only includes parameters that have a real meaning to the planner and limits the number of overcover situations. Many planners complain that schedules that have four or more work pieces overcovered are both difficult or impossible to implement in real situations and to manually change without destroying a large part of the solution.

Another important opinion obtained from our direct work with planners, is that, in some situations, it is easier to adjust a schedule with some work pieces not covered than to change a schedule with all work pieces covered as one obtains from the SCP model. The idea is working with Relaxed Models that allow some undercovered work pieces in final schedules, but obviously the number of work pieces not covered should be controlled. 
The following model description can be seen as a relaxation of the SCP $_{\text {Num_Maxc model. We }}$

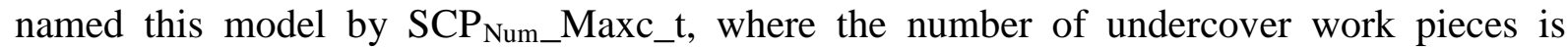
controlled by a value, $t$, defined by the user, that represents the minimum percentage of the number of work pieces that must be covered in a final schedule. Again, this parameter makes sense to the planner and he or she can define it easily based on his or her experience.

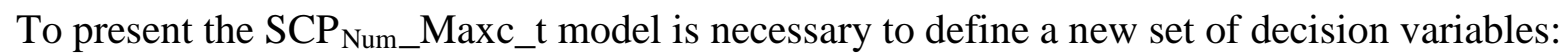

$y_{\mathrm{i}}=\left\{\begin{array}{ll}1 & \text { if the } i \text { work piece is cover by any dutie on the shedule; } \\ 0 & \text { otherwise; }\end{array}(i \in \mathrm{M})\right.$

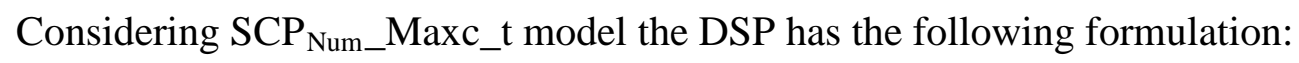

$$
\begin{array}{ll}
\left(\mathrm{SCP}_{\mathrm{Num} \_ \text {Maxc_t }}\right) \quad \text { MIN } \quad & \sum_{j=1}^{n} x_{j} \\
\text { S.a: } & y_{i} \leq \sum_{j=1}^{n} a_{i j} x_{j} \leq \operatorname{Maxc} \quad(i \in \mathrm{M}) \\
& \sum_{i=1}^{m} y_{i} \geq t \times|M| \\
& x_{j}, y_{i} \in\{0,1\} \quad(i \in \mathrm{M} ; j \in \mathrm{N})
\end{array}
$$

Constraint set (2) guarantees that all work pieces are cover by at least one and at most by Maxc duties and number constraint set (3) guarantees that the percentage of covered work pieces is higher than $t$ value.

The next and last model reflect an important comment by the planners that consider that not all work duties are equal. Depending on the type of the work piece, related to the hour and line of the passengers trips, the possibility of undercover or overcover situations can be different. Moreover in a large set of schedules it is not unusual that the overcover situation, with more then four duties covering the same work piece at the same time, occurs in the middle of the day while in relaxed models undercover work pieces occur in the beginning or end of the daily periods. To control this situation we propose an alternative approach that considers a division of $M$, work pieces set, into two subsets $M^{\mathrm{SCP}}$ and $\mathrm{M}^{\mathrm{SPP}}\left(\mathrm{M}^{\mathrm{SCP}} \cup \mathrm{M}^{\mathrm{SPP}}=\right.$ $\mathrm{M}$ and $\mathrm{M}^{\mathrm{SCP}} \cap \mathrm{M}^{\mathrm{SPP}}=\{\}$ ). The work pieces included in each subset will have different constraints related with the permission or not for overcover situations.

In this alternative model, named $\mathrm{SCP}_{\text {Num_Maxc_t_P }} \mathrm{P}_{\text {scp }}$, the subsets idea is applied to the previous $\mathrm{SCP}_{\mathrm{Num} \_}$Maxc_t model. In this approach the DSP has the following formulation: 


$$
\begin{aligned}
& \left(\mathrm{SCP}_{\text {Num_Maxc_t_P }} \mathrm{scp}\right) \quad \mathrm{MIN} \sum_{j=1}^{n} x_{j} \\
& \text { s.a: } \quad y_{i} \leq \sum_{j=1}^{n} a_{i j} x_{j} \leq \operatorname{Maxc} \quad\left(i \in \mathrm{M}^{\mathrm{SCP}}\right) \\
& \sum_{j=1}^{n} a_{i j} x_{j}=y_{i} \quad\left(i \in \mathrm{M}^{\mathrm{SPP}}\right) \\
& \sum_{i=1}^{m} y_{i} \geq t \times|M| \\
& x_{j}, y_{i} \in\{0,1\} \quad(i \in \mathrm{M} ; j \in \mathrm{N})
\end{aligned}
$$

Constraint set (2) guarantees that all $\mathrm{M}^{\mathrm{SCP}}$ work pieces, if covered, are covered by at least one and at most by Maxc duties, constraint set (3) guarantees that all $\mathrm{M}^{\mathrm{SPP}}$ work piece, if are covered, are covered only by one duty and constraints set (4) guarantees that the percentage of covered work pieces is higher than the $t$ value. Consider the value $P_{s c p}$, that represents the percentage of work pieces in the subset, $\mathrm{M}^{\mathrm{SCP}}$, i.e. $\left(\left|\mathrm{M}^{\mathrm{SCP}}\right|=\left\lfloor|M| \times P_{\text {scp }}\right\rfloor\right)$. The set $\mathrm{M}^{\mathrm{SCP}}$ can be defined by the planner or, given $P_{s c p}$, it can be generated randomly.

\section{Results}

The next four phases of the followed methodology, Verify the Model; Select the Best Alternative; Present the Results of the Analysis and Implement and Evaluate are presented in this section. As mentioned before this work is done with the full collaboration of the endusers and planners from these companies. The output schedules can be visualized by the planners and they decide about the quality and applicability.

To testing the proposed models, we selected a set of DSP instances from the main Portuguese transportation operators. The authors have a large experience with working together with several transportation operators, however for business reasons we are allowed to show only a subset of all instances seen in practice. The selected instances try to cover the entire set of different real situations found in the national passenger bus transportation business. We considered nine instances from three different transportation operators with different planning strategies. The selected instances represent the Portuguese operators. InTable I, we summarize the main characteristics of selected operators, which include:

- Urban and Suburban operators;

- Public and Private operators;

- "Family" operators that have a few known drivers and big operators with strong union force;

- Vehicle schedules with different number and duration of vehicle blocks;

- Vehicle schedules with different relief points and number of work pieces;

- Different types of duties with different rules to define them;

- Different planning strategies. 


\begin{tabular}{|c|c|c|c|c|}
\hline & & \multicolumn{3}{|c|}{ Operator } \\
\hline \multicolumn{2}{|c|}{ Properties } & CARRIS & STCP & RL \\
\hline \multirow{6}{*}{ Kind of Operator } & Urban & $*$ & $*$ & \\
\hline & Suburban & & & $*$ \\
\hline & Public & $*$ & * & \\
\hline & Private & & & * \\
\hline & Small & & & $*$ \\
\hline & Big & * & * & \\
\hline \multirow{2}{*}{ Planning Type } & "Line by Line" & * & * & \\
\hline & "By network" & & & * \\
\hline \multicolumn{2}{|l|}{ Vehicles number average } & 10 & 9 & 33 \\
\hline \multicolumn{2}{|l|}{ Relief points average } & 2 & 3 & 11 \\
\hline \multicolumn{2}{|c|}{ Relief opportunities average } & 166 & 136 & 315 \\
\hline \multicolumn{2}{|c|}{ Different duty types average } & 3 & 5 & 4 \\
\hline \multicolumn{2}{|c|}{ Planning with extra work } & No & No & Yes \\
\hline \multicolumn{2}{|l|}{ Meal break limitation } & $\begin{array}{l}\text { Lunch } \\
\text { and } \\
\text { Dinner }\end{array}$ & Lunch & No \\
\hline \multicolumn{2}{|c|}{ Obligation of start/end on the depot } & No & Yes & No \\
\hline \multicolumn{2}{|c|}{ Day periods not aloud to start/end duties } & Yes & Yes & No \\
\hline
\end{tabular}

Table I: The main characteristics of the selected operators.

Based on the selected vehicle schedules, work piece division of the vehicle blocks and the set of parameters that reflect the duty rules, all feasible duties are generated, based on the work of Agra (1993). There are three different instances for each operator covering the usual dimension of the real DSP problem that the planners should solve, see Table II.

\begin{tabular}{|l|c|c|c|c|c|c|c|c|c|}
\cline { 2 - 9 } \multicolumn{1}{c|}{} & Carr1 & Carr2 & Carr3 & STCP1 & STCP2 & STCP3 & RL1 & RL2 & RL3 \\
\hline $\boldsymbol{m}$ & 131 & 148 & 179 & 96 & 88 & 184 & 129 & 302 & 347 \\
$\boldsymbol{n}$ & 1879 & 13872 & 23747 & 2015 & 11145 & 42496 & 1839 & 5271 & 23305 \\
\hline
\end{tabular}

Table II: The dimension of the test instances.

The three proposed alternative models, $\mathrm{SCP}_{\text {Num_Maxc, }} \mathrm{SCP}_{\text {Num_Maxc_t }}$ and SCP $_{\text {Num_Maxc_t_P }}$ scp, were tested with Cplex 8.1 with parameters Maxc $=3, t=90 \%$ and $P_{s c p}=0.5$ to obtain the optimal solution for the nine instances. Cplex 8.1 is commercial software for Integer Programming Resolution from ILOG (http://www.ilog.fr/products/Cplex).

We present the summary of the results in Table III on the next two pages. Each schedule obtained by the models SPP/SCP, SPP ${ }_{\text {Num }} / \mathrm{SCP}_{\text {Num }}$ and the new proposed models $\mathrm{SCP}_{\mathrm{Num}} 3$,

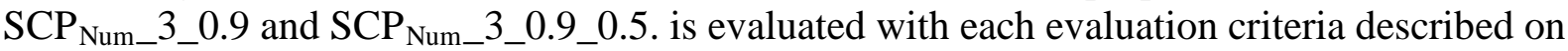
section 3. 
Recall the evaluation criteria presented in section 2:

(1) Total "real" cost;

(2) Total number of undercover pieces of work;

(3) Total duration of undercover pieces of work;

(4) Total number of overcover pieces of work;

(5) Total duration of overcover pieces of work;

(6) Maximum number of duties involved in the same overcover work piece;

(7) Unfit;

(8) Total number of duties in the optimal schedule;

(9) Number of trippers.

The shaded values on the table correspond to cases where the Cplex 8.1 was interrupted after 24 hours running without getting any final solution or models without feasible solution.

When we compare the results of the new model $\mathrm{SCP}_{\mathrm{Num} \_} 3$ with the $\mathrm{SCP}_{\text {Num }}$ model, we observe that:

- The number of duties are equal (evaluation criteria (8));

- The number of work pieces involved in overcover situations also suffers small variations (evaluation criteria (4));

- But the maximum number of duties covering the same row at the same time (evaluation criteria (6)) improved for the suburban instances (RL1,RL2 and RL3). 


\begin{tabular}{|c|c|c|c|c|c|c|c|c|}
\hline Instances & & SCP & $\mathbf{S C P}_{\mathrm{Num}}$ & SPP & $\mathbf{S P P}_{\text {Num }}$ & $\begin{array}{c}\mathrm{SCP}_{\mathrm{Num}_{-}} \\
3\end{array}$ & SCP ${ }_{\text {Num_3_0.9 }}$ & SCP $P_{\text {Num_3_0.9_0.5 }}$ \\
\hline \multirow{9}{*}{ Carr1 } & (1) & 214975 & 320966 & & & 329544 & 190392 & 181460 \\
\hline & (2) & 0 & 0 & & & 0 & 13 & 14 \\
\hline & (3) & 0 & 0 & & & 0 & 1504 & 1687 \\
\hline & (4) & 23 & 21 & & & 23 & 3 & 6 \\
\hline & (5) & 3212 & 2691 & & & 2700 & 3 & 552 \\
\hline & (6) & 4 & 4 & & & 3 & 2 & 2 \\
\hline & (7) & 37 & 29 & & & 29 & 16 & 20 \\
\hline & (8) & 38 & 38 & & & 38 & 26 & 26 \\
\hline & (9) & 3 & 3 & & & 3 & 0 & 0 \\
\hline \multirow{9}{*}{ Carr2 } & (1) & 91442 & 182148 & & & 218220 & 104036 & 104144 \\
\hline & (2) & 0 & 0 & & & 0 & 15 & 14 \\
\hline & (3) & 0 & 0 & & & 0 & 712 & 630 \\
\hline & (4) & 32 & 23 & & & 19 & 0 & 1 \\
\hline & (5) & 1551 & 1119 & & & 1008 & 0 & 53 \\
\hline & (6) & 2 & 3 & & & 3 & 1 & 2 \\
\hline & (7) & 32 & 24 & & & 21 & 15 & 14 \\
\hline & (8) & 19 & 19 & & & 19 & 14 & 14 \\
\hline & (9) & 0 & 0 & & & 0 & 0 & 0 \\
\hline \multirow{9}{*}{ Carr3 } & (1) & 117628 & 226372 & 180822 & 190398 & 226372 & 101644 & 101803 \\
\hline & (2) & 0 & 0 & 0 & 0 & 0 & 18 & 17 \\
\hline & (3) & 0 & 0 & 0 & 0 & 0 & 1187 & 1070 \\
\hline & (4) & 41 & 14 & 0 & 0 & 14 & 7 & 5 \\
\hline & (5) & 1852 & 694 & 0 & 0 & 694 & 316 & 191 \\
\hline & (6) & 3 & 2 & 1 & 1 & 2 & 2 & 2 \\
\hline & (7) & 46 & 14 & 0 & 0 & 14 & 25 & 22 \\
\hline & (8) & 24 & 22 & 22 & 22 & 22 & 17 & 17 \\
\hline & (9) & 0 & 0 & 0 & 0 & 0 & 0 & 0 \\
\hline \multirow{9}{*}{ STCP1 } & (1) & 197434 & 276164 & 197434 & 268065 & 276164 & 284391 & 284078 \\
\hline & (2) & 0 & 0 & 0 & 0 & 0 & 10 & 8 \\
\hline & (3) & 0 & 0 & 0 & 0 & 0 & 1145 & 925 \\
\hline & (4) & 0 & 6 & 0 & 0 & 6 & 3 & 0 \\
\hline & (5) & 0 & 615 & 0 & 0 & 615 & 249 & 0 \\
\hline & (6) & 1 & 2 & 1 & 1 & 2 & 2 & 1 \\
\hline & (7) & 0 & 6 & 0 & 0 & 6 & 13 & 8 \\
\hline & (8) & 25 & 21 & 25 & 21 & 21 & 17 & 17 \\
\hline & (9) & 0 & 0 & 0 & 0 & 0 & 0 & 0 \\
\hline \multirow{9}{*}{ STCP2 } & (1) & 94914 & 132791 & 94914 & 124958 & 132791 & 158199 & 158245 \\
\hline & (2) & 0 & 0 & 0 & 0 & 0 & 7 & 6 \\
\hline & (3) & 0 & 0 & 0 & 0 & 0 & 353 & 305 \\
\hline & (4) & 0 & 7 & 0 & 0 & 7 & 6 & 5 \\
\hline & (5) & 0 & 343 & 0 & 0 & 343 & 300 & 252 \\
\hline & (6) & 1 & 2 & 1 & 1 & 2 & 2 & 2 \\
\hline & (7) & 0 & 7 & 0 & 0 & 7 & 13 & 11 \\
\hline & (8) & 10 & 9 & 10 & 9 & 9 & 8 & 8 \\
\hline & (9) & 0 & 0 & 0 & 0 & 0 & 0 & 0 \\
\hline \multirow{9}{*}{ STCP3 } & (1) & & 249755 & & 264753 & 249755 & 279349 & 284119 \\
\hline & (2) & & 0 & & 0 & 0 & 18 & 19 \\
\hline & (3) & & 0 & & 0 & 0 & 959 & 997 \\
\hline & (4) & & 5 & & 0 & 5 & 2 & 2 \\
\hline & (5) & & 210 & & 0 & 210 & 100 & 100 \\
\hline & (6) & & 2 & & 1 & 2 & 2 & 2 \\
\hline & (7) & & 5 & & 0 & 5 & 20 & 21 \\
\hline & (8) & & 18 & & 18 & 18 & 15 & 15 \\
\hline & (9) & & 0 & & 0 & 0 & 0 & 0 \\
\hline
\end{tabular}


Table III: Value of the optimal schedules for the different evaluations' criteria. 


\begin{tabular}{|c|c|c|c|c|c|c|c|c|}
\hline Instances & & SCP & $\mathbf{S C P}_{\text {Num }}$ & SPP & $\mathbf{S P P}_{\mathrm{Num}}$ & $\begin{array}{c}\mathbf{S C P}_{\text {Num_- }} \\
3\end{array}$ & SCP ${ }_{\text {Num_3_0.9 }}$ & SCP ${ }_{\text {Num_}}$ 3_0.9_0.5 \\
\hline \multirow{9}{*}{ RL1 } & (1) & 297615 & 324307 & 377264 & 452913 & 312479 & 224927 & 226326 \\
\hline & (2) & 0 & 0 & 0 & 0 & 0 & 13 & 13 \\
\hline & (3) & 0 & 0 & 0 & 0 & 0 & 1633 & 1558 \\
\hline & (4) & 16 & 25 & 0 & 0 & 28 & 12 & 12 \\
\hline & (5) & 1534 & 2345 & 0 & 0 & 2845 & 917 & 896 \\
\hline & (6) & 3 & 3 & 1 & 1 & 3 & 2 & 2 \\
\hline & (7) & 20 & 29 & 0 & 0 & 33 & 25 & 25 \\
\hline & (8) & 30 & 29 & 36 & 35 & 29 & 20 & 21 \\
\hline & (9) & 1 & 2 & 5 & 11 & 1 & 0 & 0 \\
\hline \multirow{9}{*}{ RL2 } & (1) & & 893060 & & 1099083 & 928443 & 629147 & 648497 \\
\hline & (2) & & 0 & & 0 & 0 & 31 & 31 \\
\hline & (3) & & 0 & & 0 & 0 & 6977 & 6332 \\
\hline & (4) & & 65 & & 75 & 81 & 44 & 28 \\
\hline & (5) & & 6610 & & 8123 & 11273 & 3389 & 2650 \\
\hline & (6) & & 7 & & 4 & $4^{*}$ & 3 & 3 \\
\hline & (7) & & 104 & & 117 & 138 & 84 & 67 \\
\hline & (8) & & 93 & & 93 & 93 & 62 & 66 \\
\hline & (9) & & 1 & & 9 & 1 & 0 & 0 \\
\hline \multirow{9}{*}{ RL3 } & (1) & & 517158 & & & & & \\
\hline & (2) & & 0 & & & & & \\
\hline & (3) & & 0 & & & & & \\
\hline & (4) & & 36 & & & & & \\
\hline & (5) & & 2460 & & & & & \\
\hline & (6) & & 3 & & & & & \\
\hline & (7) & & 38 & & & & & \\
\hline & (8) & & 48 & & & & & \\
\hline & (9) & & 0 & & & & & \\
\hline
\end{tabular}

Table III (cont.): Value of the optimal schedules for the different evaluations’ criteria.

Apparently, the model $\mathrm{SCP}_{\text {Num_}} 3$ leads results equal to the $\mathrm{SCP}_{\text {Num }}$ model with respect to the values obtained in all evaluations criteria except number (6). However, when presenting these results to planners they comment that it is very important to have the maximum number of duties covering a piece of work under a specific value even if some other evaluation criteria increase, especially for suburban instances. Therefore, the planners find great utility in being able to obtain the solutions from both models and be able to evaluate and compare them to make a better decision. For example, in the RL2 case where evaluation criteria (6) is large for the $\mathrm{SCP}_{\text {Num }}$ model, the solution obtained by the model $\mathrm{SCP}_{\mathrm{Num}_{-} 4} 4$ is clearly better from the planners point of view, since both solutions have the same number of duties, but the maximum number of duties covering a piece of work is smaller in the solution obtained from the second model.

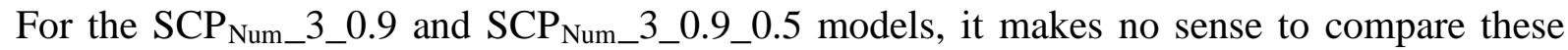
with the previous cases since these allow undercover work pieces. So, the other evaluation criteria are implicitly improved. When we make a compare them, we observe that both models have similar and balanced solutions with respect to the different evaluation criteria. We present these solutions to the planners and they commented that these schedules are easier to adjust and modify in shorter time than other schedules obtained by the previous models. They prefer to have partial solutions which are easier to adjust instead of having complete solution with too many duties and overcover situations.

\footnotetext{
* For $M a x c=3$ there is no feasible solution, so this solution was obtained for $M a x c=4$.
} 
In summary, for urban DSP instances, such as the ones from Carr and STCP, the model $\mathrm{SCP}_{\text {Num_}} 3$ works quite well, the number of overcover is under control and the solutions obtained can be implemented in real situations without a significant number of modifications. However, for the suburban instances, like the RL instances, the models SCP ${ }_{\text {Num_3_}}$ 0.9 and $\mathrm{SCP}_{\text {Num_}} 3$ _0.9_0.5 are the ones preferred by the planners. These models are very difficult to solve and being able to manually modify the schedules obtained by these models in a short period of time, is the most important characteristic noted by the planners. Therefore the new proposed models have an advantage of better applicability, with similar simplicity and quality of solutions with respect to the classical models based on SPP/SCP.

\section{Conclusions}

In this work we have considered the Driver Scheduling Problem in a realistic context. Our objective is to propose new mathematical models to this problem that can more closely represent the complexity and issues presented in the planning process of the transportation companies. The development of these models is based on a broad collaboration with different bus transportation companies in Portugal.

We followed the phases of the Operations Research Methodology to analyse and evaluate the different alternative models for the DSP. We propose several alternative models and, the corresponding optimal schedules are analysed and criticised by the end-users and planners of the transportation companies involved. The models and respective schedules were evaluated by the planners with respect to their quality which was measured by simplicity, solution quality and applicability of the models.

The main conclusion of this research work is that the proposed models are more flexible and adaptable to the different realities and evaluation criteria for each of the different transportation operators. The planners consider that the schedules obtained from the new models are more balanced with respect to the global set of evaluation criteria, and even when the schedules are not final and directly applied to the real situation, they are easier to modify and adjust.

This study reveals that the new alternative models are better from a practical point of view for the process of solving the DSP problem. We intend to continue this modelling approach to the DSP problem and continue the work already done and consider a multiobjective approach. Simultaneously, we intent to develop and implement new metaheuristic techniques to obtain a solution for the proposed models since many of the large instances can not be solved in a reasonable timeframe by general ILP software.

\section{Acknowledgements}

Thanks are due to Fundação para a Ciência e Tecnologia (SFRH/ BD / 1054 / 2000) for providing funding to support the work described in this paper (first author). Thanks are due to Ministerio de Educación y Ciencia, Spain (SEC2003-01991/ECO) for providing funding to supportthe work (second author). 


\section{References}

1) A. Agra, Relatório de Estágio, Faculdade de Ciências da Universidade de Lisboa (1993).

2) P. Chu e J. Beasley, “A Genetic Algorithm for the Set Partitioning Problem”, Imperial College, London (1995).

3) F. Hillier \& G. Lieberman, "Introduction to Operations Research”, McGraw-Hill (1995).

4) R. Portugal, "A new approach for the Drivers Scheduling Problem in Transportation Companies", Ph.D. Thesis, Department of Statistics and Operations Research, University of Lisbon, Portugal (2006).

5) A. Ravindran, D. Phillips e J. Solberg, “Operations Research: Principles and Practice”, Jonh Wiley \& Sons (1987).

6) W. Winston, “Operations Research: Applications and Algorithms”, $3^{\text {rd }}$ Ed., Duxbury Press (1994). 